Supporting information for:

\title{
Construction and Operation Costs of Wastewater Treatment and Implications for the Paper Industry in China
}

\author{
Kunyu Niu ${ }^{\text {a,b }} \quad$ Jian $\mathrm{Wu}^{\mathrm{c}, *} \quad$ Fang Yu$^{\mathrm{b}} \quad$ Jingli Guo $^{\mathrm{a}}$ \\ 1. Institute of Agricultural Economics and Development, Chinese Academy of Agricultural Sciences, \\ Beijing, China, 100081; \\ 2.Chinese Academy for Environmental Planning, Beijing, China, 100012; \\ 3.School of Environment and Natural Resources, Renmin University of China, Beijing,China, \\ 100872 .
}

The supporting information provides additional information about the following: (1) The specification on variables; (2) Data scrubbing methodology; (3) Data Descriptive Statistics; (4) One-factor analysis of variance (ANOVA); (5) Statistical Testing Results of the Model ; (6) Main wastewater treatment processes; (7) Major price index.

Table S1 Specification table of categorical variables

Table S2 Descriptive statistical results for key variables of the functions

Table S3 One-factor analysis of variance table

Table S4 Fixed asset investment price index, fuel price index of the industry and raw material price index (2007-2014)

Figure S1 Map of the distribution of eastern, middle, and western regions of China Figure S2 Normal P-P Diagram of Construction Cost and Operation Cost Figure S3 Normal P-P Diagram of $\ln ($ Construction Cost) and $\ln ($ Operation Cost) Figure S4 Residual error distribution of the fitted construction cost function and operation cost function 


\section{Variable specifications}

In our model, the design capacity of wastewater treatment for the construction cost function, the annual wastewater treatment volume for the operation cost function and the ratio of inlet concentration to outlet concentration of COD are continuous variables. The technology factor, corporate ownership factor, regional factor, and sub-sector factor are all categorical variables. For analysis purposes, categorical variables should be transformed into dummy variables. In order to avoid collinearity, if a categorical variable can be divided into $\mathrm{n}$ dummy variables (for example, the technology factor can be divided into 6 types meaning there are 6 dummy variables), only n-1 dummy variables can be set with a value of either 0 or 1 to be included in the model, while the other dummy variable is the control variable which is omitted from the model (Table S1).

Table S1: Specification table of categorical variables

\begin{tabular}{c|c|c}
\hline Categorical Variable & Variables included in the model & Control Variable \\
\hline Technology Factor & $\begin{array}{c}\text { physical technology; } \\
\text { chemical technology; } \\
\text { physicochemical technology } \\
\text { anaerobic technology } \\
\text { anaerobic-aerobic combined } \\
\text { technology }\end{array}$ & aerobic technology \\
\hline Corporate Ownership & $\begin{array}{c}\text { private enterprises; } \\
\text { foreign enterprises }\end{array}$ & state-owned ownership enterprises \\
\hline Regional Factor & $\begin{array}{c}\text { eastern region of China; } \\
\text { middle region of China }\end{array}$ & western region of China \\
\hline Sub-sector Factor & pulp manufacture & paper and paper products manufacture \\
\hline
\end{tabular}

\section{Data Scrubbing Methodology}

The National Pollution Source Census, which involves industrial water pollution, includes the construction and operation costs of wastewater treatment facilities in industrial enterprises, industrial wastewater treatment technology, types of wastewater, treatment efficiency, areas where enterprises are located and the business ownership. We pulled out the original 7,004 samples of the paper industry from the dataset, and performed data scrubbing before establishing the model.

First, we deleted the samples whereby the important variables such as the construction cost, pollution abatement operation cost, design capacity of wastewater treatment, wastewater treatment volume, or dummy variables were missing. Second, we conducted a logical test for the remaining data samples. The criterions used are as follows: (1) The ratio of inlet concentration to outlet concentration of COD cannot be less than 1; (2) Specifically, for the data samples of the construction cost function, the variables of construction cost and design capacity of wastewater treatment cannot be zero; and (3) For the data samples of the operation cost function, the variables of pollution abatement operation cost and wastewater treatment volume cannot be zero. Finally, we removed outliers from the sample using the boxplot method.

After careful data processing, our final data sample consisted of 5,935 effective samples for construction cost function and 6,065 effective samples for operation cost function.

\section{Data Descriptive Statistics Analysis}

By examining a breakdown of the data, the number of small enterprise treatment facilities made up the 
overwhelming percentage of facilities (81.2\%). 57.4\% of treatment facilities were located in eastern China; $20.3 \%$ were located in the middle regions; and $22.3 \%$ were located in the western regions ${ }^{1}$ (The distribution of the eastern, middle, and western regions of China is shown in figure S1). Facilities conducting the pulp manufacturing represented $3.4 \%$ of the total sample size, which consumed most water and created most pollution. This process, which covered only $3.2 \%$ of the gross output from the paper industry, contributed $13.7 \%$ to wastewater discharge. Among all the samples, $91.7 \%$ of treatment facilities were from private enterprises, $7.0 \%$ were from foreign enterprises, and only $1.3 \%$ belonged to state-owned enterprises. Private enterprises are classified as all domestic enterprises except for state-owned and state-controlling enterprises. Foreign enterprises are classified as China-based businesses which have been solely or partially invested in using foreign capital from outside mainland China (including from overseas, Hong Kong, Macau and Taiwan).State-owned enterprises include solely state-owned enterprises, and state-controlling enterprises.

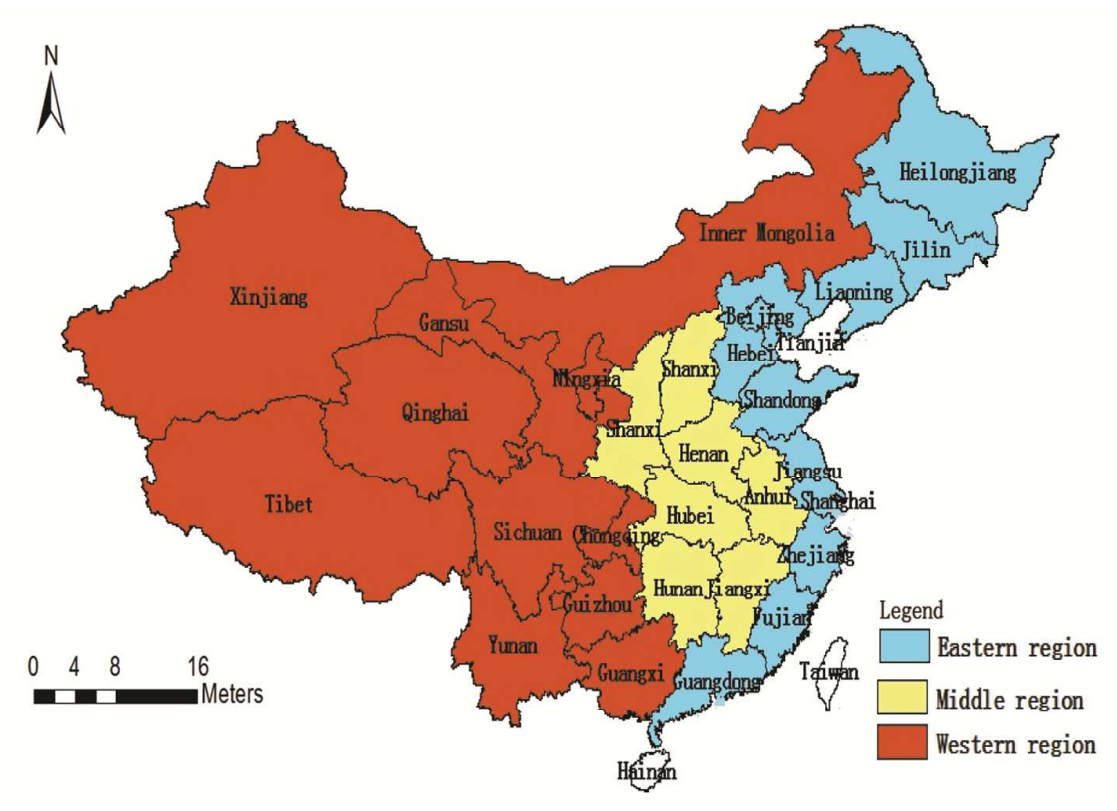

Figure S1 Distribution map of the eastern, middle, and western regions of China

Note: there is no sample data from the white-colored province.

Table S2 shows the descriptive statistical results for the four key continuous variables of construction cost, design capacity of wastewater treatment, operation cost, and wastewater treatment volume. It shows that a $1 / 4$ fractile, the median, $3 / 4$ fractile and the maximum of the sample size for the design capacity of wastewater treatment in the paper industry are $250 \mathrm{~m}^{3} / \mathrm{d}, 1,200 \mathrm{~m}^{3} / \mathrm{d}, 4,300 \mathrm{~m}^{3} / \mathrm{d}$ and $550,000 \mathrm{~m}^{3} / \mathrm{d}$ respectively; and that a $1 / 4$ fractile, the median, $3 / 4$ fractile and the maximum of the sample size for the annual wastewater treatment volume of the paper industry are $25,000 \mathrm{~m}^{3} / \mathrm{y}, 150,000 \mathrm{~m}^{3} / \mathrm{y}, 578,000 \mathrm{~m}^{3} / \mathrm{y}$ and $35,283,000 \mathrm{~m}^{3} / \mathrm{y}$ respectively. The results show that all four variables demonstrate a right skew distribution.

\footnotetext{
${ }^{1}$ The Eastern regions include: Beijing, Tianjing, Shanghai, Jilin Province, Heilongjiang Province, Hebei Province, Liaoning Province, Jiangsu Province, Zhejiang Province, Fujian Province, Shandong Province, Guangdong Province, Hainan Province; Middle regions include: Shanxi Province, Anhui Province, Jiangxi Province, Hunan Province, Hubei Province, Henan Province; Western regions include: Guangxi Province, Xinjiang, Inner Mongolia, Ningxia, Sichuan Province, Guizhou Province, Gansu Province, Tibet, Qinghai Province, Yunnan Province, Sh'anxi Province, Chongqing.(Figure S1) 
Table S2 Descriptive statistical results for key variables of the functions

\begin{tabular}{|c|c|c|c|c|}
\hline & Construction Cost & DCWT & Operation Cost & AWTV \\
\hline Unit & thousand $\$$ & $\mathrm{~m}^{3}$ per day & thousand $\$$ per year & $\mathrm{m}^{3}$ per year \\
\hline Mean value & 506.3 & 4195.1 & 113.8 & 644757.8 \\
\hline Standard error of mean value & 25.8 & 135.3 & 9.0 & 21063.5 \\
\hline Medium value & 80.0 & 1200.0 & 16.0 & 151250.0 \\
\hline Standard deviation & 2026.6 & 10668.8 & 698.6 & 1640382.0 \\
\hline Variance & 2565639 & 113800000 & 304822 & $2.7 * 10^{12}$ \\
\hline Skew & 12.9 & 23.9 & 35.4 & 7.8 \\
\hline Standard error of skew & .031 & .031 & .031 & .031 \\
\hline Kurtosis & 258.5 & 1116.4 & 1698.6 & 96.6 \\
\hline Standard error of Kurtosis & .062 & .062 & .063 & .063 \\
\hline \multirow[t]{3}{*}{ Quartile } & 24.0 & 250.0 & 4.3 & 25000.0 \\
\hline & 80.0 & 1200.0 & 16.0 & 151250.0 \\
\hline & 256.1 & 4300.0 & 64.0 & 578042.5 \\
\hline
\end{tabular}

\section{One-factor Analysis of Variance}

First, normal distribution test was conducted on the construction cost value and operation cost value of samples showing as Figure S2, which showing that they are skewness distribution. The data were changed over by logarithm transformation routine, and the normal distribution examination was performed again. The logarithm transform data showed normal distribution like figure S3 shows, which means that One-factor analysis can be conducted after logarithm transformation. The results of One-factor analysis of variance are in Table S3.
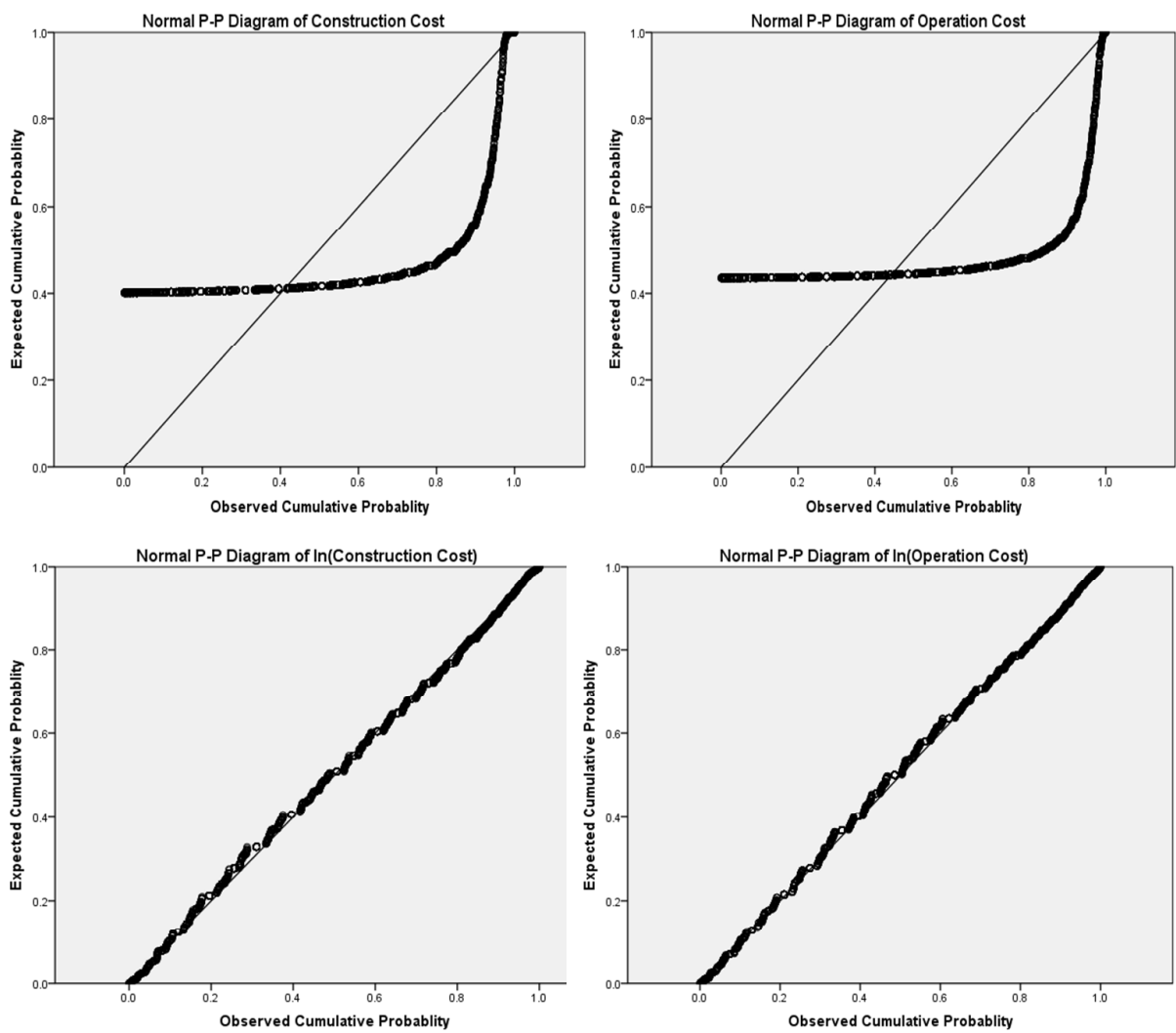

Figure S3 Normal P-P Diagram of $\ln ($ Construction Cost) and In(Operation Cost) 
Table S3 One-factor analysis of variance

\begin{tabular}{cccccc|ccccc}
\hline \multicolumn{7}{c|}{ Construction cost } & \multicolumn{5}{c}{ Operation cost } \\
\hline & Sum squares df & Meansquare & F & Sig. & Sum of squares df & Meansquare & F & Sig. \\
\hline Adjusted model & 115966.777 & 13 & 8920.521 & 3383.141 & $<0.1$ & 45701.943 & 13 & 3515.534 & $1096.236<0.1$ \\
Region & 58.503 & 2 & 29.252 & 11.094 & $<0.1$ & 335.779 & 2 & 167.889 & 52.352 & $<0.1$ \\
Sub-sector & 472.501 & 1 & 472.501 & 179.198 & $<0.1$ & 402.911 & 1 & 402.911 & $125.638<0.1$ \\
Corporate ownership & 186.571 & 2 & 93.285 & 35.379 & $<0.1$ & 130.852 & 2 & 65.426 & $20.402<0.1$ \\
Technology & 6611.125 & 7 & 944.446 & 358.185 & $<0.1$ & 5221.614 & 7 & 745.945 & $232.605<0.1$ \\
Total amount & 18011.689 & 5935 & 2.637 & & & 21906.427 & 6065 & 3.207 & & \\
Adjusted total amount & 133978.466 & 5934 & & & & 67608.370 & 6064 & & & \\
\hline
\end{tabular}

\section{Statistical Testing Results of the Model}

Figure S4 is the standardized residual histograms and P-P diagrams for construction cost function and operation cost function. It shows that the residual errors of both functions have a normal distribution, which indicates the model has a high degree of fit.
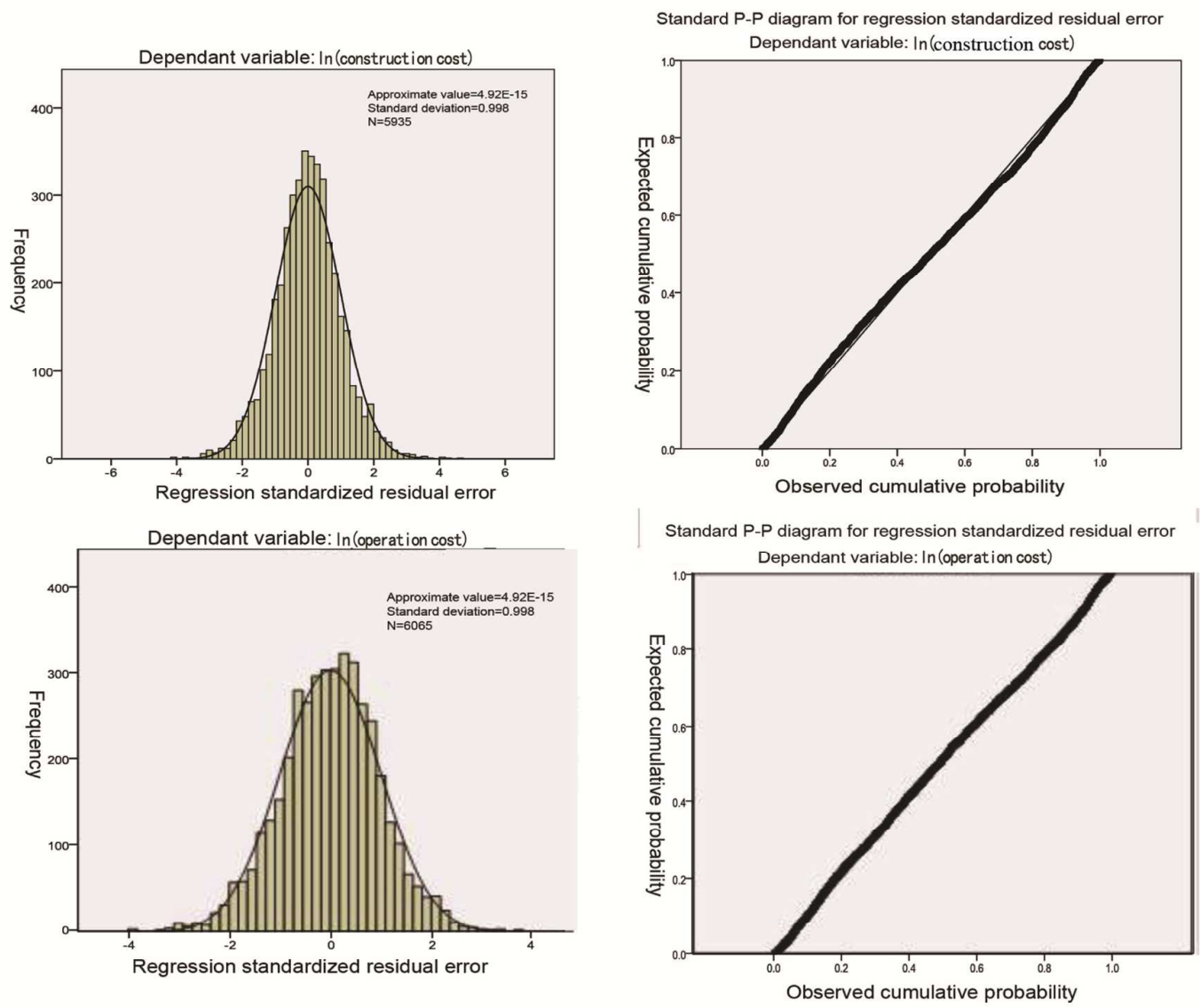

Figure S4 Residual error distribution of the fitted construction cost function and operation cost function

\section{Main wastewater treatment processes}

The wastewater treatment process in the paper industry mainly includes four types of technical processes: physical, biological, chemical, and physicochemical processes, representing 33\%, 35\%, 24\% and $8 \%$ of all 
samples respectively.

Normally, the physical process is used for primary treatment of wastewater, specifically, the removal of suspended matters in the water. The process mainly includes screening, centrifugation, sedimentation, flotation, and dissolved air flotation ${ }^{1}$. Among them, sedimentation is the most common primary wastewater treatment method ${ }^{2}$. In the UK, the method is also prioritized in the wastewater treatment of paper factories ${ }^{3}$. The statistical analysis of our samples indicates that the sedimentation method forms $68 \%$ of all physical processes.

Chemical process method includes neutralization, oxidation and reduction, and coagulation. Among them, coagulation is widely used in the wastewater treatment of the paper industry for the removal of suspended solids, color and insoluble $\mathrm{COD}^{2,4}$. In our analysis, coagulation forms $97 \%$ of all valid samples for the chemical process method. The method may be used in both primary and tertiary treatment processes. Although the pigment and suspended matters in water may be effectively removed by coagulation, the COD removal effect is less than satisfactory ${ }^{5,6}$ - some studies indicate that the COD removal rate by the coagulation method ranges from $40 \%$ to $91 \%^{7,8}$.

Biological treatment methods represent the most common secondary treatment processes, including anaerobic, aerobic, and anaerobic-aerobic combined treatment methods. Due to the ease of operation and high COD treatment rate, aerobic treatment method is most widely used in the wastewater treatment of the paper industry, ${ }^{9,10}$ covering $62 \%$ of all biological process samples. In the aerobic treatment method, the activated sludge treatment is primarily used, ${ }^{2,6}$ covering about $75 \%$ of aerobic treatment processes in the sample. The COD removal rate by aerobic treatment processes ranges from $70 \%$ to $98.4 \% .{ }^{11-18}$ Anaerobic processes are mainly used to remove high concentrations of organic wastewater. ${ }^{19}$

There are also some physicochemical treatment methods, such aselectrolysis, reverse osmosis, ultrafiltration, microfiltration, nanofiltration, ozonidation and absorption. They are normally used for tertiary treatment of wastewater and also applied in advanced treatment of wastewater for the purpose of recycling ${ }^{20}$. However, this technology has not been widely used in the paper industry.

The wastewater of the paper making industry may be divided into pulping wastewater with high concentrations of COD and wastewater from making paper and paper products (also referred to as white water) with low concentrations of COD. In most cases, it is difficult to reach the standard for wastewater discharge through a single treatment process. Processes should therefore be combined for effective wastewater treatment. For the low-concentration white water, a two-stage treatment approach may be necessary for meeting the discharge standard. For pulping wastewater, a three-stage treatment process may be necessary. Among our samples, each sample is a treatment unit process. The treatment cost for each process may be obtained through model simulation. Furthermore, the wastewater treatment cost for the combined treatment train may be obtained by adding individual treatment costs together. ${ }^{20}$ 


\section{Major Price Index}

Table S4 Fixed asset investment price index, fuel price index of the industry and raw material price index $(2007-2014)^{a}$

\begin{tabular}{c|c|c|c}
\hline & Industrial Raw Material Price Index & Industrial Fuel Price Index & Fixed Asset Investment Price Index \\
\hline 2007 & 100 & 100 & 100 \\
\hline 2008 & 108.9 & 120.6 & 108.9 \\
\hline 2009 & 100.1 & 107.6 & 106.3 \\
\hline 2010 & 110.2 & 125.1 & 110.1 \\
\hline 2011 & 120.3 & 138.6 & 117.4 \\
\hline 2012 & 117.9 & 139.9 & 119.0 \\
\hline 2013 & 114.3 & 135.1 & 119.6 \\
\hline 2014 & 110.8 & 131.2 & \\
\hline
\end{tabular}

a Data source: National Bureau of Statistics of the People's Republic of China. (2014). "China Industry Economy Statistical Yearbook." China Statistics Press: Beijing. [in Chinese].

\section{Reference:}

1. Kamali M.; Khodaparast Z. Review on recent developments on pulp and paper mill wastewater treatment. Ecotoxicology \& Environmental Safety 2015,114(4), 326-342.

2. Pokhrel, D.; Viraraghavan, T. Treatment of pulp and paper mill wastewater - a review. World Pulp \& Paper 2005, 333(1-3), 37-58.

3. Thompson G.; Swain J.; Kay M.; Forster, C. F. The treatment of pulp and paper mill effluent: a review. Bioresource Technology 2001, 77(3), 275-286.

4. Xiao L.; Sun D. Y.; Shi Y.; Han D.; Lv Y.P.; Yang D.H. Research Progress on the Treatment Technology of Regenerated Papermaking Wastewater. Water Treatment Technology, 2016, (1), 20-25.

5. Mansour, L.B.; Ksentini, I.; Elleuch, B. Treatment of wastewaters of paper industry by coagulation-electroflotation. Desalination 2007, 208(1-3), 34-41.

6. Ashrafi O.; Yerushalmi L.; Haghighat F. Wastewater treatment in the pulp-and-paper industry: A review of treatment processes and the associated greenhouse gas emission. Journal of Environmental Management 2015, 158(8), 146-157.

7. Li R. Q., Xin X. Y. (2007). “Coagulation treatment of wastewater from reproduced pulp papermaking [J].” Water Treatment Technology, 33(5), 52-54.

8. Ahmad AL.; Wong S.S.; Teng T.T.; and Zuhairi A. Improvement of alum and PACl coagulation by polyacrylamides (PAMs) for the treatment of pulp and paper mill wastewater. Chemical Engineering Journal 2008, 137(3), 510-517.

9. Bennett G. F. Environmental Biotreatment Technologies for Air, Water, Soil, and Wastes. Journal of Hazardous Materials, 2002, 93(2), 253-255. 
10. Hong, M. Y.; Li, Q. B.; Deng, X. Progress of Anaerobic(Hydrolytic)-Aerobic Combined Processes for Biological Treatment of wastewater. Environmental Protection of Chemical Industry 2005, 25(2), 104-109.

11. Junna J.; and Ruonala S. Trends and guidelines in water pollution control in the Finnish pulp and paper industry. Tappi Journal 1991, 74(7), 5-11.

12. Shi F.Y. Design and Debug on Regenerated Papermaking Wastewater Treatment Project. Environmental Science and Technology 2010, 33(7), 168-172.

13. Al-Shididi S.; Henze M.; Ujang Z. Feasibility study of sequencing batch reactor system for upgrading wastewater treatment in Malaysia. Water Science \& Technology 2003, 48(11-12), 327-35.

14. Magnus E.; Carlberg G. E.; and Norske H. H. TMP wastewater treatment including a biological high-efficiency compact reactor. Nordic Pulp and Paper Research Journal 2002, 15(1), 29-36.

15. Hansen, E.; Zadura L.; Frankowski, S.; Wachowicz, M. Upgrading of an activated sludge plant with floating biofilm carriers at Frantschach Swiecie S.A. to meet the new demands of year 2000. Water Science \& Technology 1999, 40(11), 207-214.

16. Lerner, M.; Stahl, N.; Galil, N.I. Comparative Study of MBR and Activated Sludge in the Treatment of Paper Mill Wastewater. In Forest Industry Wastewaters VIII.; IWA Publishing: London, United Kingdom 2007; pp 23-29.

17. Mahmood, T.; Paice, M. Aerated stabilization basin design and operating practices in the Canadian pulp and paper industry. Journal of Environmental Engineering \& Science 2006, 5(5), 383-395.

18. Norris, P.; Marshall, R.; Richard, M. High temperature effects on activated sludge treatment performance and sludge quality in a recycle mill. In Proceedings of the 2000 TAPPI International Environmental Conference and Exhibit. Technical Assoc. of the Pulp and Paper Industry Press: Denver, United states 2000; pp 383-406.

19. Buzzini, A. P.; Gianotti, E.P.; Pires, E.C. UASB performance for bleached and unbleached kraft pulp synthetic wastewater treatment. Chemosphere 2005, 59(1), 55-61.

20. Plumlee M H.; Stanford B D.; Debroux, J. F.; Hopkins, D. C.; Snyder, S. A. Costs of Advanced Treatment in Water Reclamation. Ozone Science \& Engineering 2014, 36(5), 485-495. 